\title{
Synthesis and Characterization of Novel Functional Electrosterically Stabilized Colloidal Particles Prepared by Emulsion Polymerization Using a Strongly Ionized Amphiphilic Diblock Copolymer
}

\author{
P. S. Mohanty, ${ }^{*, \perp, \dagger}$ H. Dietsch, ${ }^{\dagger}$ L. Rubatat, ${ }^{\ddagger, \#}$ A. Stradner, ${ }^{\dagger}$ K. Matsumoto, ${ }^{\S, \prime \prime}$ \\ H. Matsuoka, ${ }^{\S}$ and P. Schurtenberger ${ }^{\dagger}$ \\ Adolphe Merkle Institute and Fribourg Centre for Nanomaterials and Department of Physics and Fribourg \\ Centre for Nanomaterials, University of Fribourg, 1700 Fribourg, Switzerland, Department of Polymer \\ Chemistry, Kyoto University, Kyoto 615-8510, Japan, and Molecular Engineering Institute, Kinki \\ University, 11-6 Kayanomori Iizuka Fukuoka Prefecture 820-8555, Japan
}

\begin{abstract}
Amphiphilic diblock copolymers such as poly(styrene)-block-poly(styrene sulfonate) (PS- $b$-PSS) (Matsuoka, H.; Maeda, S.; Kaewsaiha, P.; Matsumoto, K. Langmuir 2004, 20, 7412), belong to a class of new polymeric surfactants that ionize strongly in aqueous media. We investigated their self-assembly behavior in aqueous solutions and used them as an emulsifier to prepare electrosterically stabilized colloidal particles of different diameters between 70 to $400 \mathrm{~nm}$. We determined the size, size polydispersity, effective charge, total dissociable charge, structural ordering, and phase behavior using light scattering, transmission electron microscopy (TEM), small-angle neutron scattering (SANS), and potentiometric titration. These experiments clearly demonstrated that all of the synthesized particles were nearly monodisperse (polydispersity index $\leq 6 \%$ ). The results of DLS and TEM clearly suggested the existence of hairy particles. The form factors obtained by SANS were well described by a polydisperse sphere model. The estimated total number of dissociable charges per particle was found to be larger than $10^{4} e$, whereas the effective charges per particle were found to be around $1000 e$. This significant difference suggested the confinement of charges inside the corona regions of the polyelectrolyte brush shell. Finally, these monodisperse particles were found to self-assemble into $3 \mathrm{D}$ ordered colloidal crystalline arrays at a low volume fraction $(=0.00074)$ that diffract light in the visible region.
\end{abstract}

\section{Introduction}

Colloidal dispersions serve as an excellent model system in condensed matter physics because of their importance in fundamental ${ }^{1-4}$ studies as well as in high-tech applications such as Bragg diffraction devices, ${ }^{5}$ nanoswitches,${ }^{6}$ chemical sensors, ${ }^{7}$ and templates for preparing photonic band gap materials. ${ }^{8}$ These

* Corresponding author. E-mail: pmohanty@pc.rwth-aachen.de.

† Adolphe Merkle Institute and Fribourg Centre for Nanomaterials, University of Fribourg.

* Department of Physics and Fribourg Centre for Nanomaterials, University of Fribourg.

\& Kyoto University.

"Kinki University.

${ }^{\perp}$ Current address: Institute of Physical Chemistry II, RWTH Aachen University, Landoltweg 2, 52056 Aachen, Germany.

${ }^{*}$ Current address: EPCP/IPREM UMR 5254, Unìversity de Pau, 64000 Pau Cedex, France.

(1) Russell, W. B. Colloidal Dispersions; Cambridge University Press: New York, 1989.

(2) Mohanty, P. S.; Tata, B. V. R.; Yamanaka, J.; Sawada, T. Langmuir 2005, 21,11678 .

(3) Matsuoka, H.; Yamamoto, T.; Harada, T.; Ikeda, T. Langmuir 2005, 21, 7105.

(4) Mohanty, P. S.; Kesavamoorthy, R.; Matsumoto, K.; Matsuoka, H.; Venkatesan, K. A. Langmuir 2006, 22, 4552.

(5) Guisheng, P.; Kesavamoorthy, R.; Asher, S. A. Phys. Rev. Lett. 1999, 78, 3860

(6) Asher, S. A.; Guisheng, P.; Kesavmoorthy, R. Nonlinear Opt. 1999, 21, 343.

(7) Marta, K.; Igor, k. L.; Alexander, M.; Kesavmoorthy, R.; Asher, S. A. Adv. Funct. Matter 2003, 13, 774.

(8) Asher, S. A.; Serban, P.; Reese, E.; Xiang, L.; David, F. Anal. Bioanal. Chem. 2002, 373, 632 . colloidal dispersions can be either sterically stabilized ${ }^{9,10}$ or electrostatically stabilized. ${ }^{11-13}$ Usually, steric stabilization can be provided by nonionic amphiphilic block copolymers whereas electrostatic stabilization can be provided by classical lowmolecular-weight surfactants such as sodium dodecyl sulfate (SDS). Both sterically stabilized and electrostatically stabilized colloidal systems have been extensively studied in the past.

Colloidal systems that combine both steric and charge stabilization are called electrosterically ${ }^{14}$ stabilized colloids. They offer considerable advantages over the other two types of colloidal systems. Here, the interparticle interactions can be tuned using several parameters compared to conventional colloidal systems, and they are expected to show high stability against increasing salt concentration. In the present work, we used strongly ionic amphiphilic diblock copolymers as an emulsifier in emulsion polymerization to prepare electrosterically stabilized colloidal particles. Amphiphilic block copolymers exhibit unique properties in emulsion polymerization, owing to their low critical micelle concentration $(\mathrm{cmc})$ and low diffusion coefficient compared to

(9) van Megen, W; Pusey, P. N. Nature 1986, 320, 340.

(10) Pusey, P. N.; van Megen, W. Phys. Rev. Lett. 1987, 59, 2083.

(11) Tata, B. V. R.; Mohanty, P. S.; Valsakumar, M. C. Phys. Rev. Lett. 2004, 88,018302 .

(12) Sirota, E. B.; Ou-Yang, H. D.; Sinha, S. K.; Chaikin, P. M.; Axe, J. D.; Fujji, Y. Phys. Rev. Lett. 1989, 62, 293.

(13) Yamanaka, J.; Yoshida, H.; Koga, T.; Ise, N.; Hashimoto, T. Phys. Rev. Lett. 1998, 80, 5806.

(14) Napper, D. H. Polymeric Stabilization of Colloidal Dispersions; Academic Press: New York, 1983. 
that of classical low-molecular-weight surfactants. ${ }^{15}$ Furthermore, in the case of ionic amphiphilic block copolymers, it is also possible to control both the steric and charge stabilization by simply controlling the hydrophilic and hydrophobic block lengths of the block copolymer chains. Although there are many studies reporting on the application of nonionic block copolymers in emulsion polymerization, there are only a few studies on the preparation of colloidal particles using ionic amphiphilic diblock copolymers $^{16-20}$ and triblock copolymers. ${ }^{21}$ For example, Leemans et al. ${ }^{16}$ used poly(alkyl methycrylate-block-sulfonated glycidyl methacrylate) for the synthesis of polymer acrylic latex particles. Müller et al. ${ }^{17}$ used poly(ethyl ethylene)-blockpoly(styrenesulfonate) as a stabilizer for emulsion polymerization. Rager et al. ${ }^{18}$ investigated the aggregates formed by poly(acrylic acid)-block-poly(methyl methacrylate) block copolymers and their applicability as stabilizers in emulsion polymerization. Bouix et al. ${ }^{19}$ and Wang et al. ${ }^{20}$ reported initial studies on poly(styrene)block-poly(styrene sulfonate) as a stabilizer in the preparation of colloidal particles. The applications of both nonionic and ionic block copolymers in emulsion polymerization have been described in a recent review by Riess et al. ${ }^{22}$ However, to our knowledge, a detailed study on the synthesis and characterization of monodisperse charged colloidal particles of different diameters using a strongly ionized poly(styrene)-block-poly(styrene sulfonate) amphiphilic block copolymer does not exist.

Usually, anionic amphiphilic diblock copolymers are much more difficult to synthesize because of the large difference in solubility of each block as compared to that of cationic polymers, and there have been only a few studies on this topic. ${ }^{23-25}$ However, Matsuoka et al. $^{26}$ recently synthesized anionic amphiphilic diblock copolymers containing a sulfonic group. These block copolymers were strongly ionized in aqueous media. ${ }^{26-29}$ Moreover, they formed almost monodisperse micelles above a critical block copolymer concentration (the critical micelle concentration, $\mathrm{cmc}$ ), and these micelles were stable against high salt concentrations. Because of these properties, this novel class of polymeric surfactants seems to be an ideal candidate for the preparation of monodisperse colloidal particles via emulsion polymerization. We thus started to take advantage of these polymers in order to synthesize particles with different diameters. Previously, ${ }^{30}$ we reported preliminary results on the conductivity and potentiometric titration of the obtained particles, which showed very curious titration profiles indicating anomalous

(15) Förster, S.; Zisenis, M.; Wenz, E.; Antonietti, M. J. Chem. Phys. 1996, $104,9956$.

(16) Leemans, L.; Fayt, R.; Teyssie, Ph.; de Jaeger, N. C. Macromolecules 1991, 24, 5922

17) Müller, H.; Leube, W.; Tauer, K.; Forster, S.; Antonietti, M. Macromolecules 1997, 30, 2288 .

(18) Rager, T.; Meyer, W. H.; Wegner, G.; Mathauer, K.; Machtle, W.; Schrof, W.; Urban, D. Macromol. Chem. Phys. 1999, 200, 1681.

(19) Bouix, M.; Gouzi, J.; Charleus, B.; Vairon, J.; Guinot, P. Macromol. Rapid Commun. 1998, 19, 209.

(20) Wang, L.; Chuang, W. R.; Chiu, W. Y.; Cheng, K. C. Polym. Prepr. (Am. Chem. Soc., Div. Polym. Chem.) 2002, 43, 297.

(21) Walther, A.; Hoffmann, M.; Müller, Axel H. E. Angew. Chem., Int. Ed. 2008, 47, 711 .

(22) Riess, G.; Labbe, C. Macromol. Rapid Commun. 2004, 25, 401.

(23) Astafieve, I.; Khougaz, K.; Eisenberg, A. Macromolecules 1995, $28,7127$.

(24) Gabaston, L. L.; Furlong, S. A.; Jackson, R. A.; Armes, S. P. Polymer 1999, 40, 4505 .

(25) Förster, S.; Hermsdorf, N.; Leube, W.; Schnablegger, H.; Linder, P.; Böttcher, C. J. Phys. Chem. B 1999, 103, 6657.

(26) Matsuoka, H.; Maeda, S.; Kaewasaiha, P.; Matsumoto, K. Langmuir 2004, 20,7412 .

(27) Matsuoka, H.; Matsutani, M.; Mouri, E.; Matsumoto, K. Macromolecules $\mathbf{2 0 0 3}, 36,5321$.

(28) Kaewasaiha, P.; Matsumoto, K.; Matsuoka, K. Langmuir 2007, 23, 9162 (29) Matsuoka, H.; Furuya, Y.; Kaewsaiha, P.; Mouri, E.; Matsumoto, K. Macromolecules 2007, 40, 766.

(30) Mohanty, P. S.; Harada, T.; Matsumoto, K.; Matsuoka, H. Macromolecules 2006, 39, 2016
Table 1. Characteristics of PS- $b$-PSS Block Copolymers ${ }^{a}$

\begin{tabular}{cccc}
\hline$m^{b} / n^{c}$ & $M_{\mathrm{n}}{ }^{b}$ & $M_{\mathrm{w}} / M_{\mathrm{n}}{ }^{b}$ & degree of sulfonation ${ }^{c}$ \\
\hline $90: 65$ & 20000 & 1.3 & 0.95
\end{tabular}

${ }^{a} m$ : degree of polymerization of the hydrophobic block (PS). $n$ : degree of polymerization of the hydrophilic block (PSS). $\mathrm{M}_{n}$ : number-average molecular weight. $M_{\mathrm{w}} / M_{\mathrm{n}}$ : polydispersity index. ${ }^{b}$ Determined by GPC with THF as an eluent. ${ }^{c}$ Determined by ${ }^{1} \mathrm{H}$ NMR.

dissociation behavior of the polyelectrolyte brush chains around the particle core. In this article, we present details of our studies on the self-assembly behavior of the block copolymers in aqueous solution. We demonstrate the use of these polymers as an emulsifier to prepare particles with different diameters using styrene as a monomer. Finally, we describe the characterization of thus-synthesized colloidal particles with respect to their size, size polydispersity, total dissociable charge, effective charge, and phase behavior.

The article is organized as follows. In the Experimental Section, we present details regarding the synthesis of the colloidal particles and summarize all of the experimental methods used in the present study. The Results and Discussion section is divided into three sections. In section one, we investigate the self-assembly behavior of PS- $b$-PSS block copolymers in aqueous media using static and dynamic light scattering. In section two, the characterization of all synthesized particles is described using dynamic light scattering, transmission electron microscopy (TEM), small-angle neutron scattering (SANS), and titration. In section three, we investigate the structural ordering and phase behavior of these particles in gaslike, liquidlike, and crystalline states using static and dynamic light scattering.

\section{Experimental Section}

Block Copolymer Synthesis. Poly(styrene)-block-poly(styrenesulfonate) (PS- $b$-PSS) was synthesized using the procedure reported by Okamura et al. ${ }^{31}$ The characteristics of the PS- $b$-PSS block copolymers are summarized in Table 1. Details of the synthesis procedure of PS- $b$-PSS block copolymers have been reported in a previous publication by Matsuoka et al. ${ }^{26}$

Block copolymer solutions of PS- $b$-PSS were prepared first by dissolving the block copolymers in $\mathrm{THF}$-water mixtures (50:50) and then evaporating the organic solvent (THF) slowly using a rotary vacuum evaporator to obtain block copolymer solutions in pure water. Finally, appropriate concentrations of aqueous block copolymer solutions were prepared for the light scattering studies as well as for the synthesis of colloidal particles.

Synthesis of Colloidal Particle. Colloidal particles were synthesized via emulsion polymerization with PS- $b$-PSS as the emulsifier and styrene as the monomer. Before starting the synthesis, the polymerization inhibitors were removed by washing the monomer three times with $1 \mathrm{~N} \mathrm{NaOH}$ solution and then once again with Milli-Q water. Potassium peroxodisulfate $\left(\mathrm{K}_{2} \mathrm{~S}_{2} \mathrm{O}_{8}\right)$ was used as an initiator for the polymerization.

A typical recipe for the emulsion polymerization can be described as follows: Polymerization was performed in a $300 \mathrm{~mL}$ three-necked reactor (usually a round-bottomed flask) immersed in a thermostatted water bath and equipped with a reflux condenser (water cooling), an argon inlet, and a thermometer. Initially, the reactor containing block copolymer solutions ( concentration $=0.0005 \mathrm{~g} / \mathrm{mL}$ ) was purged with Ar gas for 20 to $30 \mathrm{~min}$. Then, $7 \mathrm{~g}$ of styrene monomer was added to these block copolymer solutions under stirring, and the mixture was heated to $70{ }^{\circ} \mathrm{C}$ under $\mathrm{Ar}$ gas with proper water circulation. The temperature of the water bath was maintained at 70 $( \pm 2)^{\circ} \mathrm{C}$. Finally, an aqueous solution of the initiator $\left(\mathrm{K}_{2} \mathrm{~S}_{2} \mathrm{O}_{8}, 0.15 \mathrm{~g}\right.$ dissolved in $10 \mathrm{~mL}$ of deionized water) was added to the mixture to start the polymerization. The polymerization was stopped after

(31) Okamura, H.; Takatori, Y.; Tsunooka, M.; Shirai, M. Polymer 2002, 43, 3155 . 
Table 2. Characteristics of Synthesized Colloidal Particles ${ }^{a}$

\begin{tabular}{cclccrrr}
\hline samples & $\mathrm{BC}(\mathrm{g}) / \mathrm{MN}(\mathrm{g})$ & $D_{h}(\mathrm{PD})(\mathrm{nm})$ & $D_{\mathrm{TEM}}(\mathrm{nm})$ & $D_{\text {SANS }}(\mathrm{nm})$ & $Z_{\text {tot }}(e)$ & $\sigma_{\text {chain }}\left(\mathrm{nm}^{-2}\right)$ & $Z_{\text {eff }}(e)$ \\
\hline S1 & 0.014 & $95(4.0 \%)$ & $72 \pm 6$ & $74.9(5 \%)$ & $2.0 \times 10^{4}$ & 0.017 \\
S2 & 0.01 & $158(2.5 \%)$ & $139 \pm 3$ & $140.5(3 \%)$ & $1.4 \times 10^{5}$ & 0.037 \\
S3 & 0.0055 & $264(2.0 \%)$ & $244 \pm 2.5$ & $251(1.5 \%)$ & $6.6 \times 10^{4}$ & 0.005 \\
S4 & 0.004 & $341(20.0 \%)$ & $322 \pm 5$ & $324(5.5 \%)$ & $3.0 \times 10^{5}$ & 0.014
\end{tabular}

${ }^{a} D_{\mathrm{h}}$ : hydrodynamic diameter determined by DLS. $D_{\text {TEM }}$ : average diameter obtained by transmission electron microscopy. $D_{\text {SANs }}$ diameter obtained by small-angle neutron scattering. PD: polydispersity index. $Z_{\mathrm{tot}}$ : total dissociable charges obtained from titration. $Z_{\text {eff: }}$ effective charges measured by conductivity versus dilution experiment. $\sigma_{\text {chain }}$ : chain density (no. of chains per unit area of the particle). BC/MN: ratio of block copolymer (g) to monomer (g).

$24 \mathrm{~h}$ to ensure complete conversion. After the polymerization was finished, the reaction mixture was slowly cooled to room temperature, and the obtained dispersion was passed through a glass filter to remove the coagulum part.

Different diameters of particles (70 to $400 \mathrm{~nm}$ ) were prepared in a similar way by varying the ratio of block copolymer to monomer concentration (Table 2). After the synthesis, these suspensions were dialyzed with Milli-Q water (low conductivity water, $<1 \mu \mathrm{S}$ ) for 3 weeks to remove unreacted monomers and ionic impurities. Finally, mixed bed ion-exchange resins were added to the dialyzed suspensions to keep the suspensions fully deionized, and then they were stored in a refrigerator.

Solubilization Experiment. Hydrophobic dye solubilization experiments were carried out to evaluate the $\mathrm{cmc}$ using oil orange $\mathrm{SS}\left(\mathrm{C}_{17} \mathrm{H}_{14} \mathrm{~N}_{2} \mathrm{O}\right)$ with only visual observation.

Specific Refractive Index Increment Measurements $(\mathrm{d} n / \mathrm{d} C)$. The refractive index increment $\mathrm{d} n / \mathrm{d} C$ was determined using a differential refractometer (DRM 3000, Otsuka Electric, Japan) and accompanying software at a wavelength of $632.8 \mathrm{~nm}$. Block copolymer solutions of four to five concentrations (ranging from 0.005 to $0.0005 \mathrm{~g} / \mathrm{mL}$ ) were prepared for the $\mathrm{d} n / \mathrm{d} C$ measurements. The value of $\mathrm{d} n / \mathrm{d} C=0.245 \pm 0.005 \mathrm{~mL} / \mathrm{g}$ was determined from the slope of a plot of refractive index versus polymer concentration.

Potentiometric (pH) Titration. $\mathrm{pH}$ titrations were carried out to determine the total dissociable charges for all of the synthesized colloidal particles with different diameters. These titrations were carried out using a Metrohm 665 autotitrator (Switzerland) equipped with an argon inlet and a temperature controller. The colloidal suspensions were equilibrated for $30 \mathrm{~min}$ before the start of the titration. The titrations were performed at $25^{\circ} \mathrm{C}$.

For the effective charge measurements, conductivity was recorded as a function of particle concentration at a temperature of $25^{\circ} \mathrm{C}$.

Light Scattering. Light scattering studies were carried out with a DLS-7000 system (Otsuka) at a wavelength of $632.8 \mathrm{~nm}$. For these studies, very low sample concentrations were used to avoid a measurable influence from interparticle interactions.

Theory of Static Light Scattering. We determined the radius of gyration $\left(R_{\mathrm{g}}\right)$ and the weight-average molecular weight $\left(M_{\mathrm{w}}\right)$ of the micelles by static light scattering from the scattering angle $(\theta)$ and concentration $(C)$ dependences of the intensity of the scattered light. Under the condition of weak particle-particle interactions, the relationship among $M_{\mathrm{w}}, R_{\mathrm{g}}$, and $C$ can be expressed by ${ }^{32}$

$$
\frac{K C}{R(\theta)}=\left(\frac{1}{M_{\mathrm{w}}}+2 A_{2} C\right)\left(1+\frac{1}{3} R_{\mathrm{g}}{ }^{2} Q^{2}\right)
$$

where $K$ is an optical constant $\left(=2 \pi n_{\mathrm{o}}^{2}((\mathrm{~d} n) /(\mathrm{d} C))^{2} /\left(\lambda^{4} N_{\mathrm{A}}\right)\right), C$ is the polymer concentration in $\mathrm{g} / \mathrm{mL}, A_{2}$ is the second viral coefficient, which is a measure of intermicellar interaction, $Q=(4 \pi n) /\left(\lambda_{\mathrm{o}}\right) \sin$ $(\theta / 2)$ is the scattering wave vector, $n_{\mathrm{o}}$ is the refractive index of the solvent, $\mathrm{d} n / \mathrm{d} C(=0.245 \mathrm{~mL} / \mathrm{g})$ is the refractive index increment, $\lambda$ is the wavelength of the laser light used, and $N_{\mathrm{A}}$ is Avogadro's number. $R(\theta)$, the Raleigh ratio, which is a measure of the absolute scattering intensity at scattering angle $\theta$, is calculated from the observed scattered intensity using $R(\theta)=R_{90}{ }^{\text {tol }}\left(\left(n_{\mathrm{o}}\right) /\left(n_{\text {toluene }}\right)\right)^{2}$

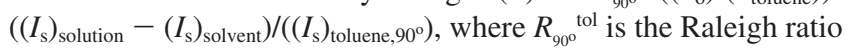
of toluene at $90^{\circ}$ and $I_{\mathrm{s}}$ is the scattering intensity.

(32) Huglin, M. B., Ed. Light Scattering from Polymer Solutions; Academic Press: New York, 1972.
Theory of Dynamic Light Scattering. The normalized intensity autocorrelation function $g^{(2)}(Q, t)$ was measured with dynamic light scattering (DLS), and the field autocorrelation function, $g^{(1)}(Q, t)$, was extracted using the Siegert relation. Then, $g^{(1)}(Q, t)$ was analyzed by cumulant analysis using ${ }^{33}$

$$
\ln \left[g^{(1)}(Q, t)\right]=-\Gamma t+\frac{\mu_{2} t^{2}}{2}
$$

where $\Gamma$ is the characteristic decay rate and $\mu_{2}$ is the second-order cumulant coefficient that is related to the degree of polydispersity by $\mu_{2} / \Gamma^{2}$. $\Gamma$ is related to the translational free diffusion coefficient by $D_{\mathrm{o}}=\Gamma / Q^{2}$. The use of DLS and eq 2 yields in general the socalled collective diffusion coefficient, which in the limit of negligible interaction effects corresponds to the ideal or free diffusion coefficient $D_{\mathrm{o}}$ and is thus related to the hydrodynamic radius $\left(R_{\mathrm{h}}\right)$ through the Stokes-Einstein equation, $D_{\mathrm{o}}=k_{\mathrm{B}} T / 6 \pi \eta R_{\mathrm{h}}$, where $k_{\mathrm{B}}$ is the Boltzmann constant, $T$ is the absolute temperature, and $\eta$ is the viscosity of the solvent.

Three-Dimensional Light Scattering. The structural ordering and phase behavior of colloidal suspensions (i.e., the presence of gaslike, liquidlike, and crystalline suspensions and their structural and dynamic properties) were investigated with a 3D light scattering instrument based on a cross-correlation technique ${ }^{34}$ (LS instruments, Switzerland) at a wavelength of $632 \mathrm{~nm}$ in order to efficiently suppress any contribution from multiple scattering. For the $3 \mathrm{D}$ light scattering measurements, we used cylindrical $10 \mathrm{~mm}$ quartz cells. Briefly, in a 3D cross-correlation experiment, two laser beams were focused on a single scattering volume, and two detectors were used to perform two scattering experiments with identical scattering vectors in exactly the same scattering volume. The individual scattering signals were cross correlated, and the cross-correlation function $G_{12}(t)$ was obtained. ${ }^{34}$ Under these conditions, $G_{12}(t)$ is then given by

$$
G_{12}(Q, t) \approx I_{1} I_{2}+\beta_{12} I_{1}^{(1)} I_{2}^{(1)}\left|g^{(1)}(Q, t)\right|^{2}
$$

where $g^{(1)}(Q, t)$ corresponds to the field autocorrelation function measured in the absence of multiple scattering. $I_{1}$ and $I_{2}$ are the time-averaged intensities measured at detectors 1 and 2 , and $I_{1}{ }^{(1)}$ and $I_{2}{ }^{(1)}$ are the corresponding scattering-intensity contributions from singly scattered light only. The 3D measurements then also provide a measure of the scattering intensity free of multiple scattering $I^{(1)}(Q)$ through

$$
I^{(1)}(Q)=\left(I_{1}(Q) I_{2}(Q) \beta_{12} / \beta_{\text {ref }}\right)^{0.5}
$$

$\beta_{12}$ is the measured intercept of the correlation function, and $\beta_{\text {ref }}$ is the intercept measured under ideal conditions in the absence of multiple scattering (i.e., with a very dilute reference sample). The details of the method are given elsewhere. ${ }^{34}$

Small-Angle Neutron Scattering (SANS). SANS measurements for dilute samples $(C=0.0044 \mathrm{~g} / \mathrm{mL})$ were carried out to determine the form factors of our synthesized particles. These experiments were performed at the SANS I facility at the Swiss spallation neutron source, SINQ, Paul Scherrer Institute, Villigen, Switzerland. We used $1 \mathrm{~mm}$ Hellma quartz cells with a thermostatted sample holder.

(33) Brown, W., Ed. Dynamic Light Scattering; Oxford Science Publication, 1993.

(34) Urban, C.; Schurtenberger, P. J. Colloid Interface Sci. 1998, 207, 150. 


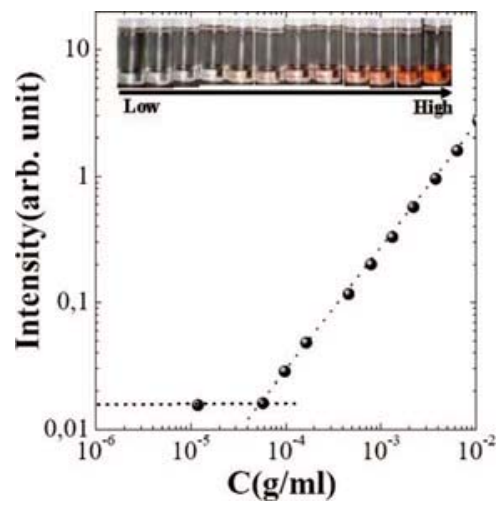

Figure 1. Relative scattering intensity at $90^{\circ}$ plotted as a function of block polymer concentration. The inset shows photographs of different block copolymer solutions with an added hydrophobic dye as a function of increasing polymer concentration.

The temperature of the samples was maintained at $25^{\circ} \mathrm{C}$ during the measurement. A sample-to-detector distance of $20.27 \mathrm{~m}$, a collimation length of $18 \mathrm{~m}$, and a wavelength of $12.67 \AA$ combined with neutron lenses allowed us to access a $q$ range of $0.006-0.2 \mathrm{~nm}^{-1}$. The raw spectra were corrected for background from the solvent $\left(\mathrm{H}_{2} \mathrm{O}\right)$, sample cell, and electronic noise by conventional procedures. Furthermore, the $2 \mathrm{D}$ isotropic scattering spectra were corrected for detector efficiency by dividing by the incoherent scattering spectra of pure water and were azimuthally averaged.

Transmission Electron Microscopy. Bright-field imaging was performed on a CM100 Philips TEM operated at $80 \mathrm{kV}$ (emission 2). All images were acquired on an SIS Morada CCD camera. Copper grids covered with a carbon film were used to deposit the particle suspension ( $c=0.0005 \mathrm{~g} / \mathrm{mL}$ ). The solvent was slowly evaporated (over $12 \mathrm{~h}$ minimum) at room temperature.

\section{Results and Discussion}

Self-Assembly Behavior of PS- $b$-PSS Block Copolymers in Aqueous Solution. The self-assembly behavior of PS- $b$-PSS block copolymers in an aqueous medium was investigated using static and dynamic light scattering. The inset of Figure 1 shows photographs of block copolymer solutions as a function of increasing polymer concentration $(C)$ with an added waterinsoluble hydrophobic dye. This Figure clearly indicates that above a certain polymer concentration the solution was colored by the dye and the color became more prominent with increasing polymer concentration. This can be attributed to the formation of polymer micelles, where the hydrophobic dye molecules are solubilized in the hydrophobic core of the micelles. We also verified the formation of micelles by measuring the relative scattering intensity as a function of block copolymer concentration. Figure 1 shows that above a polymer concentration of about $6.0 \times 10^{-5} \mathrm{~g} / \mathrm{mL}$ the intensity increases. This indicates that larger structures (e.g., micelles) have formed.

The inset of Figure 2A shows a linear dependence of $K C / R(\theta)$ on $\sin ^{2}(\theta / 2)$ at a concentration of $0.00049 \mathrm{~g} / \mathrm{mL}$. This is a clear indication of the presence of nearly monodisperse micellar aggregates. By fitting with eq 1 and by extrapolating to zero angle, $K C / R(\theta=0)$ as well as an apparent radius of gyration $R_{\mathrm{g}, \text { app }}$ was obtained. Similarly, we obtained $\operatorname{KClR}(\theta=0)$ at different concentrations (between $6.0 \times 10^{-5}$ and $0.005 \mathrm{~g} / \mathrm{mL}$ ), and its concentration dependence is shown in Figure 2A. It can be seen clearly that $K C / R(\theta=0)$ versus concentration is linear over the entire concentration range investigated, with a very small slope. From the $y$ intercept and the slope, we can then determine the weight-average molecular weight, $M_{\mathrm{w}}$, and $A_{2}$ using eq 1. Extrapolating $R_{\mathrm{g}, \text { app }}$ to $C=0$ also provides us with the
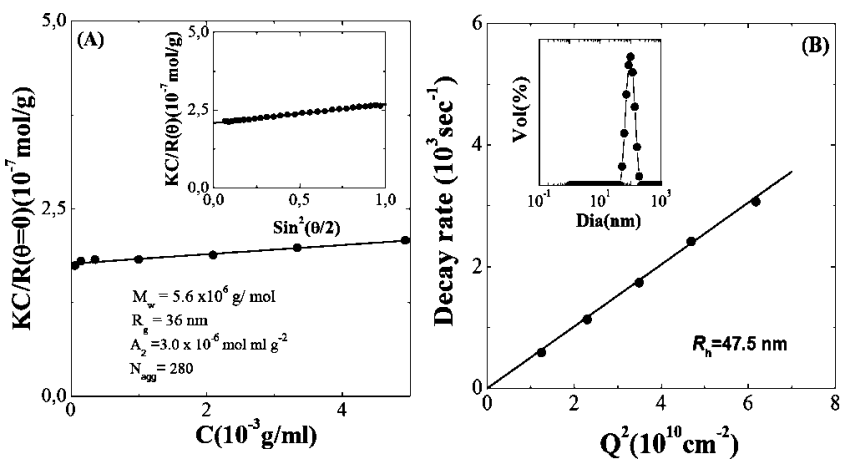

Figure 2. (A) $K \mathrm{C} / R(\theta=0)$ plotted as a function of different block copolymer concentrations. From the linear fit, the averaged molecular weight, $M_{\mathrm{w}}$, and the second virial coefficient, $A_{2}$, were obtained. The inset shows $\sin ^{2}(\theta / 2)$ as a function of $K C / R(\theta)$ at a concentration of $C$ $=0.00049 \mathrm{~g} / \mathrm{mL}$. (B) Decay rate $(\Gamma)$ plotted as a function of the square of the scattering wave vector $Q^{2}$. From the slope, the free diffusion coefficient $\left(D_{\mathrm{o}}\right)$ was obtained.

z-average radius of gyration $R_{\mathrm{g}}$. The values for $M_{\mathrm{w}}$ and $R_{\mathrm{g}}$ of the block copolymer micelles are found to be $5.6 \times 10^{6} \mathrm{~g} / \mathrm{mol}$ and $36 \mathrm{~nm}$, respectively. Furthermore, we estimated the aggregation number, $N_{\mathrm{agg}}$, from the ratio of $M_{\mathrm{w}}$ of the micelles to that of individual polymers. $N_{\text {agg }}$ is found to be 280 . Because of the large hydrophobic PS block, the size of block copolymer micelles formed by a diblock copolymer is higher than that of micelles formed by traditional low-molecular-weight surfactants. Previous studies using SANS and SAXS by Matsuoka et al. ${ }^{26}$ on PS- $b$ PSS spherical block copolymer micelles have yielded values for $N_{\text {agg }}$ of about 90 even for a considerably smaller hydrophobic PS block (degree of polymerization $=54$ ). It was also mentioned in a review by Tuzar et. al. ${ }^{35}$ that typical block copolymer micelle molecular weights have values in the range of $10^{5}$ to $10^{8} \mathrm{~g} / \mathrm{mol}$, with corresponding aggregation numbers between 10 and 1000 and micellar dimensions of up to $100 \mathrm{~nm}$ depending on molecular weight.

The second viral coefficient, $A_{2}$, provides information about the interactions between micelles. In our case, $A_{2}$ is found to be very low, around $10^{-6} \mathrm{~mol} \mathrm{~mL} / \mathrm{g}^{2}$. This low value of $A_{2}$ indicates that the interactions between the micelles are very weak and that the electrostatic contributions to the intermicellar interactions are effectively screened.

Information about the hydrodynamic radius $\left(R_{\mathrm{h}}\right)$ of the micelle was obtained using dynamic light scattering (DLS) at a very dilute concentration $(=0.00049 \mathrm{~g} / \mathrm{mL})$, where static light scattering experiments have already demonstrated the absence of a measurable influence from intermicellar interactions. Therefore, these experiments directly yield the $z$-average free diffusion coefficient and provide information about the micellar size distribution. Figure 2B shows the dependence of the decay rate $\Gamma$ on $Q^{2}$. The linear dependence and the fact that the fit passes through the origin confirm that the measured correlation function contains contributions from purely diffusive processes, reflect the translational diffusion of the micelles, and also indicate a narrow size distribution. The inset of Figure 2B shows the corresponding size distribution of the micelles obtained from an inverse Laplace transform of the correlation function using CONTIN. ${ }^{33}$ From the slope of the curve $\Gamma$ versus $Q^{2}$, the average free diffusion coefficient, $D_{0}$, is obtained, from which we calculated the corresponding $z$-average hydrodynamic radius, $R_{\mathrm{h}}$. The polydispersity of our polymer micelles determined from

(35) Tuzar, Z.; Kratochvil, P Micelles of Block and Graft Copolymers in Solution. In Surface and Colloid Science; Matjevic, E., Ed.; Plenum Press: New York, 1993; Vol 15, Chapter 1, p 1. 

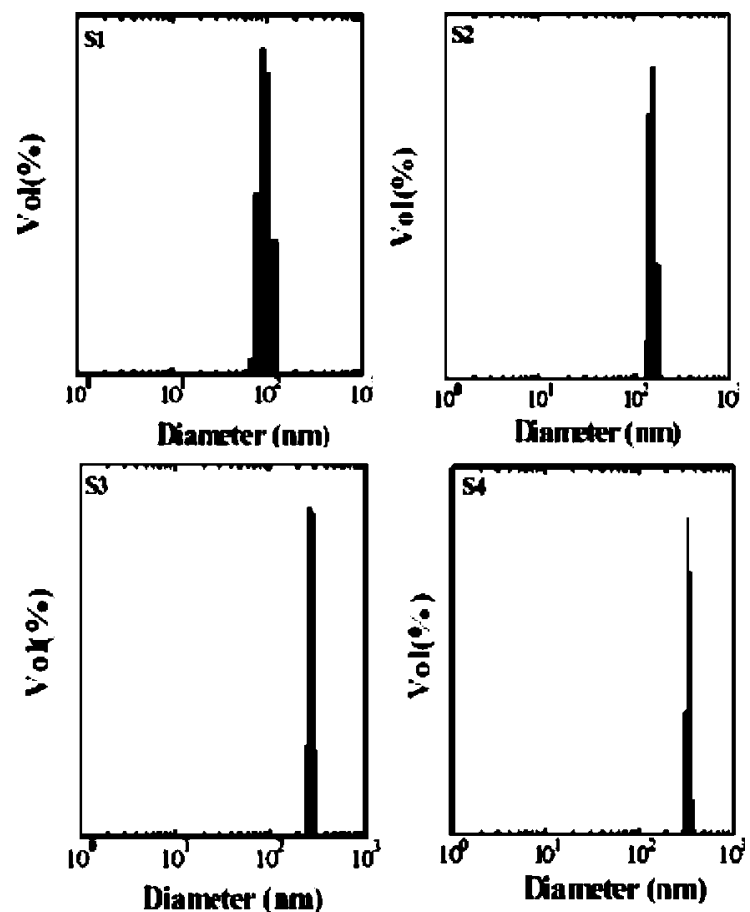

Figure 3. Plots of the volume-weighted particle size distributions for suspensions $\mathrm{S} 1-\mathrm{S} 4$ as determined from a CONTIN analysis of the results from dynamic light scattering measurements.

cumulant analysis is less than $10 \%$. In the next step, we can compare $R_{\mathrm{g}}$ and $R_{\mathrm{h}}$ and calculate the ratio $R_{\mathrm{g}} / R_{\mathrm{h}}$ as a characteristic parameter indicative of the overall micellar architecture. For homogeneous and compact spherical particles, $R_{\mathrm{g}} / R_{\mathrm{h}}$ has a value of 0.78 . In our case, we obtain a value of 0.75 , which is compatible with a spherical shape and a compact structure with a dense PSS shell around the PS core. Within the ranges of concentration of block copolymers investigated in our present study, $R_{\mathrm{g}} / R_{\mathrm{h}}$ remains the same.

Characterization of Colloidal Particles. Four different kinds of colloidal particles (S1, S2, S3, and S4) with diameters between 70 and $400 \mathrm{~nm}$ were subsequently synthesized by emulsion polymerization as described in the Experimental Section. The synthesized colloidal particles were extensively characterized using DLS, TEM, SANS, and titration as follows.

Particle Size Measurement. The hydrodynamic radius $\left(R_{\mathrm{h}}\right)$ was estimated by DLS in a very dilute suspension $(0.00005$ $\mathrm{g} / \mathrm{mL}$ ). The dilution was made to ensure that the particles do not experience measurable interaction effects. The samples prepared for light scattering were thoroughly passed through a $0.45 \mu \mathrm{m}$ filter (for S1 and S2) and a $0.8 \mu \mathrm{m}$ filter (for S3 and S4) before measurement. $R_{\mathrm{h}}$ and the polydispersity index were obtained by cumulant analysis, and the full size distribution was determined using CONTIN. ${ }^{33}$ Figure 3 shows the corresponding size distribution obtained for suspensions $\mathrm{S} 1-\mathrm{S} 4$. The Figure shows that all four suspensions possess a narrow size distribution. Table 2 summarizes the results from these measurements. The polydispersity of all synthesized particles was found to be below $6 \%$, further confirming that the synthesis resulted in the formation of nearly monodisperse particles. In addition to DLS, we also performed TEM as well as SANS studies to further confirm these data and to obtain additional information about the particle architecture.

TEM micrographs showed the corresponding colloidal particles (from S1 to S4) to be spherical and very monodisperse (Figure 4). A comparison between DLS and TEM indicates that the diameters obtained by TEM (Table 2 ) were systematically smaller
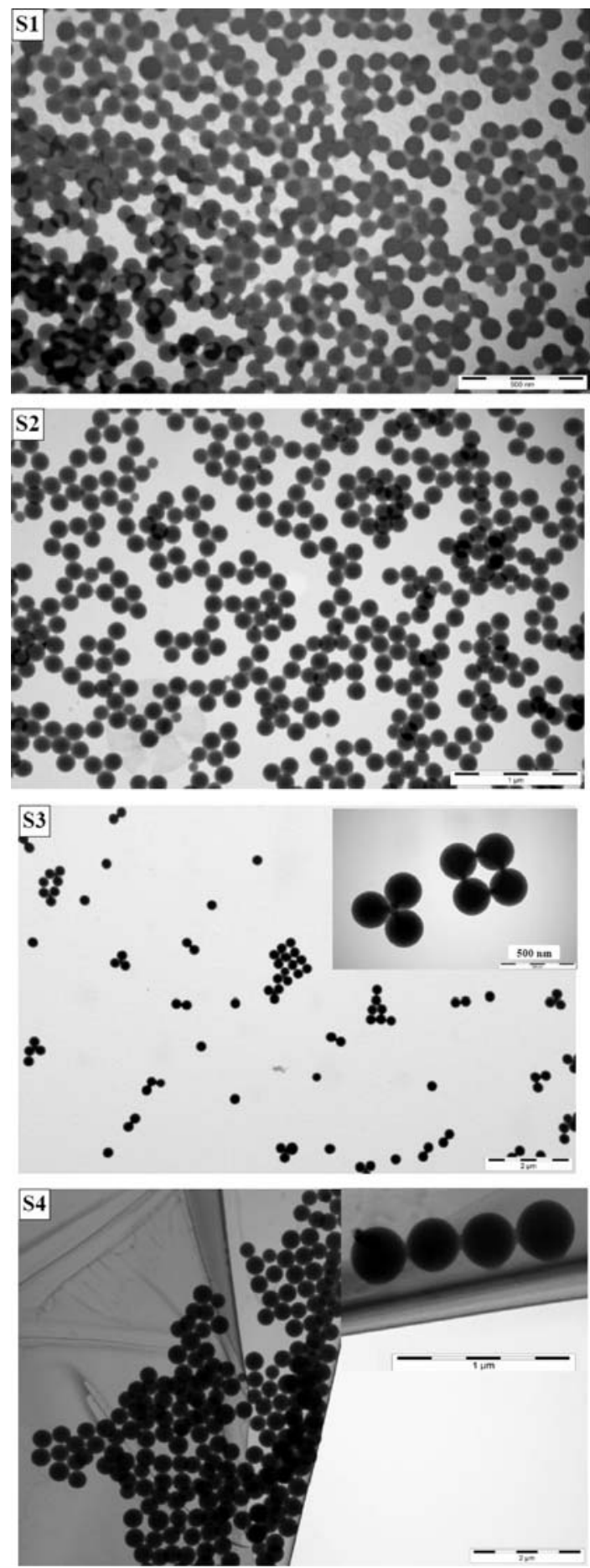

Figure 4. Corresponding TEM micrograph of particles in a dried state for S1, S2. S3, and S4. The scale bars for S1, S2, S3, and S4 are 500 $\mathrm{nm}, 1 \mu \mathrm{m}, 2 \mu \mathrm{m}$ (inset, $500 \mathrm{~nm}$ ), and $2 \mu \mathrm{m}$ (inset, $1 \mu \mathrm{m}$ ), respectively.

than those obtained by DLS, with the largest difference for the smallest particles. The likely cause of this discrepancy can be found in the different preparation states and the different mechanisms on which the two methods are based. DLS measurements were made at high dilution and low salt content, where the PSS shell is in a highly extended conformation, whereas the TEM studies were carried out on dried samples where we expect a collapsed state of the PSS chains. Recent studies on PS- $b$-PSS colloidal particles prepared by photopolymerization ${ }^{36}$ have shown that in a suspension state at low salt concentrations the PSS shell of PS- $b$-PSS colloidal particles is indeed in a highly 

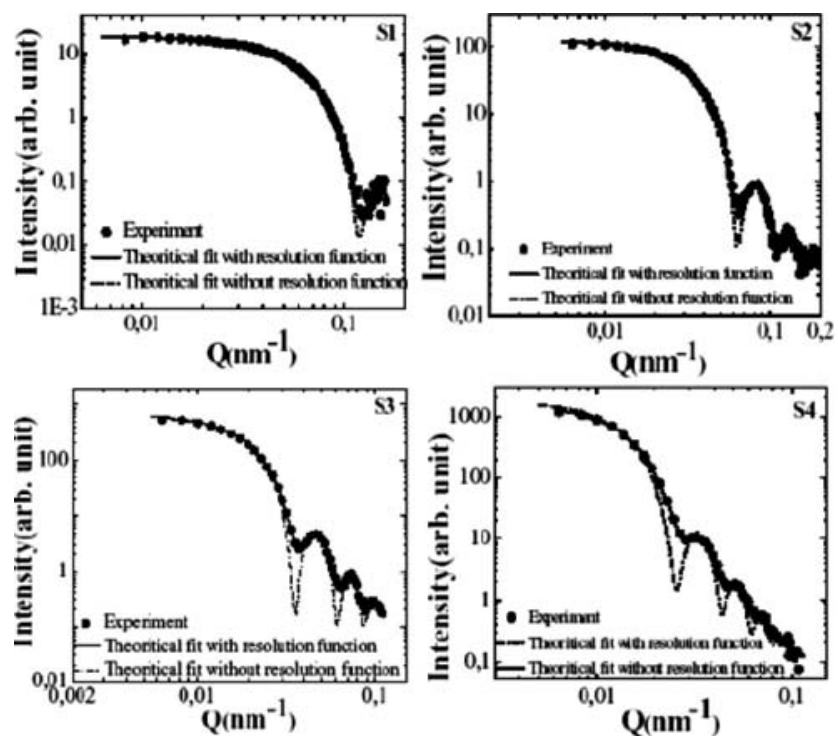

Figure 5. Scattering intensity vs scattering vector obtained from SANS measurements with suspensions $\mathrm{S} 1-\mathrm{S} 4$. The concentration of the suspension was kept at $0.0044 \mathrm{~g} / \mathrm{mL}$ with a salt content of $0.5 \mathrm{mM}$.

swollen state and that there are two major contributions to this extensive swelling of the polyelectrolyte corona observed under these conditions. First, the hydrophilic PSS polymer chain attains a stretched conformation as a result of strong electrostatic interactions among the ionic groups present on the PSS chains. Second, the counterions are strongly confined inside the polyelectrolye corona. In our case, we also expect that the PSS chains are in a stretched conformation, and the diffusion coefficient measured with DLS then reflects the overall size of the core-shell particles, which thus leads to an increase in the hydrodynamic radius, $R_{\mathrm{h}}$, compared to the value obtained from TEM for a particle with a collapsed shell in the dried state (Table 2 ). These results clearly suggested evidence of formation of hairy colloidal particles. Similar behavior has also been reported in hairy PS-PEO latex particles. ${ }^{22}$ In contrast to the study performed by Gao and Ballauff, ${ }^{36}$ where a much longer PSS block length has been used, the smaller hydrophilic block length of our PSS chain does not allow us to observe the core-shell structure in the TEM micrographs.

Additional detailed information on the particle size, shape, and architecture was obtained from SANS measurements with relatively dilute samples, which allowed us to determine the effective particle form factor $P(Q)$. Furthermore, information on the interparticle interactions and the resulting structural properties with more concentrated samples was also obtained by SANS. Here, the effective static structure factor, $S(Q)$, was extracted from the scattering intensity, $I(Q)$, through the relationship $I(Q) / C$ $\propto M_{\mathrm{w}} P(Q) S(Q)$. SANS measurements for the determination of $P(Q)$ were carried out for suspensions $(\mathrm{S} 1-\mathrm{S} 4)$ at $0.0044 \mathrm{~g} / \mathrm{mL}$ containing $0.5 \mathrm{mM} \mathrm{NaCl}$ salt. The salt was added to the suspension in order to effectively screen the electrostatic interactions between the charged colloidal particles. In this case, the structure factor approaches unity $(S(Q) \approx 1)$, and hence $I(Q) / C \propto P(Q)$. Figure 5 shows the scattering intensity $I(Q)$ versus $Q$ for $\mathrm{S} 1-\mathrm{S} 4$. A first look at Figure 5 clearly shows the sharp, distinct maxima and minima for the four different colloids that are typical of spherical particles with a narrow size distribution.

We can now further analyze our experimental data using an explicit model based on the TEM and DLS experiments in order to obtain quantitative information about the particle size and the

(36) Gao, X.; Ballauff, M. Phys. Rev. E 2001, 64, 051406. polydispersity. ${ }^{37,38}$ Here we applied a model of polydisperse homogeneous spheres where we also incorporated the appropriate instrument resolution function in order to include the smearing effect induced by the instrumental setup. A Schulz distribution was used to describe the polydispersity of the particles. The details of the procedure for the data analysis are given elsewhere. ${ }^{37}$ The solid lines in Figure 5 are the corresponding fitted curves for this model to the experimental data, which are in very good agreement with the data for all four suspensions. Note the considerable effect of the instrument resolution function that is also shown in Figure 5 as the dashed lines. The obtained diameters and the corresponding values for the size polydispersity are given in Table 2. The polydispersity is in close agreement with the results from DLS. One may notice that the diameters obtained by SANS are smaller than those obtained by DLS and closer to those obtained by TEM. This can be explained as the result of the combination of two effects: (1) For the SANS measurements that were made at higher concentrations, we added salt to the suspensions in order to avoid the presence of any measurable structure factor due to electrostatic repulsions. However, this electrostatic screening may also lead to a corona consisting of polymer chains that are now less extended and thus to a correspondingly smaller overall diameter of the particles. (2) Because of the weak scattering contrast of the corona for neutrons, the scattering of the PS core is dominating and thus also determining the size of the particles. Thus, we expect the diameter determined by SANS to be close to the diameter measured by TEM in a dried state. Furthermore, the quantitative agreement between the fitted and measured data over the entire $Q$ range, in particular, at low $Q$ values, where repulsive electrostatic interactions would lead to a reduction of the forward scattering, indicates the absence of measurable structural correlations between the colloidal particles in suspension as a result of the effective screening of electrostatic interactions between particles by the added salt.

Measurement of the Total Dissociable Surface Charges $\left(Z_{t o t}\right)$ and Effective Charges $\left(Z_{\text {eff }}\right)$. For a complete characterization of PS- $b$-PSS colloidal particles, it is essential to know the total dissociable charges due to the presence of strong sulfonic groups. This total dissociable surface charge was determined by potentiometric titration. Initially, all of the suspensions were made basic ( $\mathrm{pH}>10.0$ ) by adding $5 \mathrm{~mL}$ of $0.01 \mathrm{~N} \mathrm{NaOH}$ and were kept overnight to reach equilibrium conditions. Then these suspensions were titrated with $0.01 \mathrm{~N} \mathrm{HCl}$. A Gran plot ${ }^{39}$ was constructed by plotting $V_{\mathrm{HCl}} \times 10^{-\mathrm{pH}}$ versus $V_{\mathrm{HCl}}$ (Figure 6). The linear portions were fitted with a straight line. From the $X$-axis intercept, the excess $\mathrm{OH}^{-}$concentration was obtained for the suspension, from which we determined the total number of dissociable charges per particle. From the total number of dissociable charges, we estimated the chain density of the particle. These are summarized in Table 2 for all of the suspensions (S1 to $\mathrm{S} 4)$. The total dissociable charge $\left(\mathrm{Z}_{\mathrm{tot}}\right)$ is more than $10^{4} e$ for all of the synthesized particles. These high values of total dissociable charges suggest that the synthesized particles are highly charged. However, at the same time it is also known that these $Z_{\text {tot }}$ values are not directly related to the electrostatic interparticle interactions. ${ }^{1,4}$ Because of charge renormalization effects such as counterion condensation, we instead have to look at the so-called effective charges ${ }^{4}\left(Z_{\text {eff }}\right)$. To measure these effective charges, conductivity was recorded as a function of particle concentration (or volume fraction). From the slope of the

(37) Zackrisson, M.; Stradner, A.; Schurtenberger, P.; Bergenholtz, J. Langmuir 2005, 21, 10835 .

(38) Pedersen, J. S. Adv. Colloid Interface Sci. 1997, 70, 171

(39) Gran, G. Acta Chem. Scand. 1950, 4, 559. 

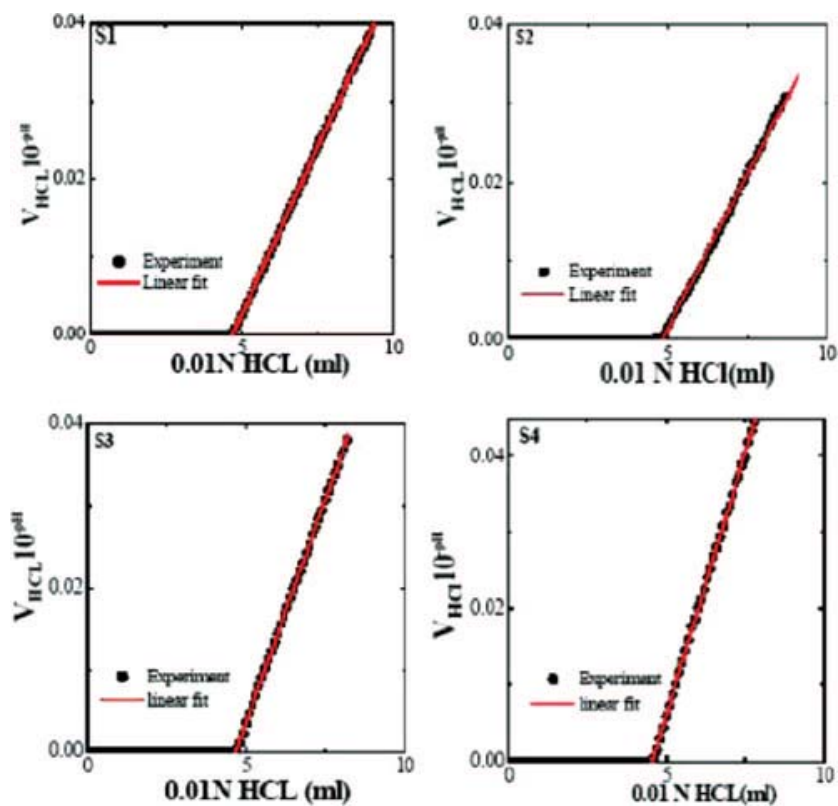

Figure 6. Gran plots for titrated suspensions $\mathrm{S} 1-\mathrm{S} 4$. The linear region is fitted to a straight line that intercepts the $X$ axis. From the intercept, the excess $\mathrm{OH}^{-}$concentration was determined, and from that, the total number of dissociable charges per particles was estimated. Concentrations of S1, S2, S3, and S4 at which the titrations were carried out are 0.0036 , $0.002,0.0063$, and $0.00588 \mathrm{~g} / \mathrm{mL}$, respectively.

conductivity versus volume fraction curve, $Z_{\text {eff }}$ was calculated using the following formula, ${ }^{4} \sigma_{e}=$ slope $\times\left(N_{\mathrm{A}}\right) /\left(\lambda_{\mathrm{H}^{+}}\right) \times(e d) /(6)$ Coulomb $/ \mathrm{cm}^{2}$. Here, $\sigma_{e}$ is the effective charge density $\left(=Z_{\text {eff }} e / \pi d^{2}\right), N_{\mathrm{A}}$ is Avogadro's number, $\lambda_{\mathrm{H}^{+}}\left(=349.8 \mathrm{~S} \mathrm{~cm}^{2}\right.$ $\mathrm{mol}^{-1}$ at $25^{\circ} \mathrm{C}$ ) is the equivalent conductance of $\mathrm{H}^{+}$at infinite dilution, $e$ is the electronic charge $\left(=e=1.6022 \times 10^{-19} \mathrm{C}\right)$, and $d$ is the diameter of the particle. The number of effective charges was found to be around $1000 e$ for all of the suspensions (Table 1) and thus significantly lower than the total number of charges. This enormous difference between $Z_{\text {tot }}$ and $Z_{\text {eff }}$ clearly showed that most of the charges were confined to the area near the particle surface (i.e., in the corona). This confinement effect of charges inside the polyelectrolyte shell has also been observed in other polymer brush systems prepared by photopolymerization. $^{36}$

Structural Ordering and Phase Behavior. In the next step, we focused on structural ordering and the phases present in these colloidal suspensions as a function of concentration using 3D static and dynamic light scattering. These studies were performed with suspensions prepared by particles with a diameter of $d=$ $158 \mathrm{~nm}$ (sample S2) because all of the important features of the scattering curves are then covered by the $Q$ range accessible with light scattering. To facilitate a comparison of our data with previous experimental and theoretical work, we now use volume fractions $(\phi)$ as the unit of concentration. $\phi$ was estimated using the corresponding mass concentration and the density of the colloidal particles $\left(=1.05 \mathrm{~g} / \mathrm{cm}^{3}\right)$.

First we investigated suspensions at very low concentrations $(\phi \approx 0.00003)$ where we expect negligible interparticle interactions even in the absence of added salt. At this low volume fraction, the average interparticle separation between the colloidal particles is large, and hence the electrostatic interactions are very weak. The structure factor $S(Q)$ was obtained from the measured scattering intensity $I(Q)$ divided by the form factor $P(Q)$ after proper concentration normalization. Figure 7A shows that $S(Q)$ is independent of $Q$. This featureless $S(Q)$ suggests that the particles are spatially uncorrelated and that the suspension has a structure that resembles gaslike order. ${ }^{4}$ Dynamic information from this gaslike suspension is obtained from the field correlation function $g^{(1)}(Q, t)$ at different values of $Q$. For noninteracting particles, the field correlation function can be written as ${ }^{4}$ $g^{(1)}(Q, t)=\exp \left(-D_{0} Q^{2} t\right)$. In a monodisperse gaslike suspension, correlation functions $g^{(1)}(Q, t)$ measured at different $Q$ positions are expected to overlap when normalized with $Q^{2}$. This is shown in Figure 7B, where all of the curves at different values of $Q$ are found to superimpose completely. This further confirmed that particles are essentially noninteracting at a very low volume fraction, $\phi \approx 0.00003$.

For suspensions at higher volume fractions, we expect to find significant positional correlations due to strong electrostatic interactions among the charged PS- $b$-PSS colloidal particles. Several samples prepared with volume fractions between 0.00005 and 0.005 showed pronounced liquidlike order within several days of preparation. Upon complete removal of any residual ions through an ion-exchange resin, crystallization and the formation of extended colloidal crystals can be observed for volume fractions as low as 0.00074 in our suspensions. It is important to realize that the ion-exchange process is very slow and may still proceed after several weeks, depending upon the concentraton of ionic impurities initially present. Here we studied the structural and dynamic processes in suspensions that exhibit either liquidlike or crystalline order.

Figure $8 \mathrm{~A}$ shows the typical signature of a suspension that exhibits liquid order at a volume fraction of 0.001 . The structure factor $S(Q)$ shows well-pronounced first- and second-order peaks and a less-pronounced third-order peak. This suggests a highly ordered liquidlike structure very close to crystallization, further supported by the height of the first peak that is close to the value of 2.8 where crystallization usually sets in. For strongly correlated particle suspensions, the dynamic properties described by the dynamic structure factor $S_{\mathrm{d}}(Q, t)$ no longer exhibit simple $Q^{2}$ scaling but also follow the static structure factor. Therefore, the different field autocorrelation functions $g^{(1)}(Q, t)$ obtained with a suspension possessing liquidlike order do not fall on each other when normalized with $Q^{2}$ (Figure $8 \mathrm{~B}$ ). At $Q=Q_{\max }$, DLS provides a measure of the self-diffusion coefficient that is considerably slowed down for strongly repulsive suspensions on the length scale of the average particle-particle distance. At $Q \gg Q_{\max }$, DLS monitors the local motion of the particles that is much less hindered on length scales significantly smaller than the typical interparticle distance where the colloids can be viewed essentially as free particles. At $Q \ll Q_{\max }$, DLS provides a measure of the collective motion of particles, which for repulsive particles is significantly enhanced. However, it is important to point out the clear indication of the strongly nonexponential behavior of $g^{(1)}(Q$, $t$ ) under these conditions. Here we observe the influence from the very small but non-negligible polydispersity. For highly correlated suspensions where the forward scattering is strongly reduced, this results in a measurable incoherent contribution to the static structure factor at low values of $Q$ that then results in an additional self-diffusive component in $g^{(1)}(Q, t)$ that becomes visible as an additional slow decay. Such complex dynamics at different $Q$ values have been previously reported as the hallmark of strongly correlated particle suspensions as a result of longrange electrostatic repulsions. ${ }^{4,40,41}$

Additional insight into the dynamics of such strongly correlated suspensions can be gained by looking at the initial decay of the correlation function $g^{(1)}(Q, t)$ at different scattering vectors. From

(40) Pusey, P. N. J. Phys. A: Math. Gen. 1978, 11, 119.

(41) Gruner, F.; Lehmann, W. P. J. Phys. A: Math. Gen. 1982, 15, 2847. 

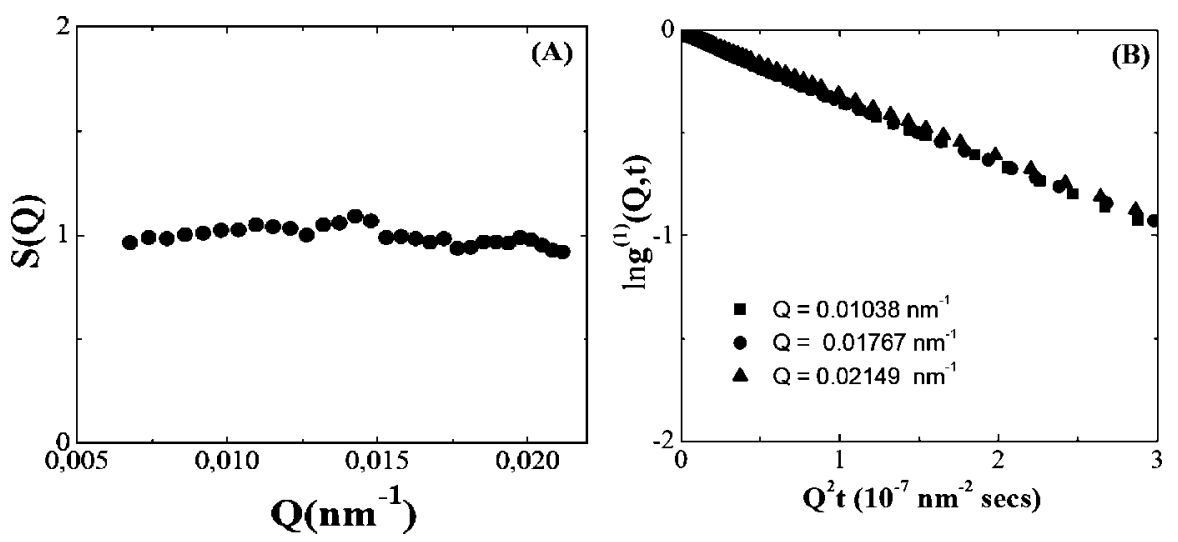

Figure 7. (A) $S(Q)$ versus $Q$ from static light scattering for a suspension with volume fraction $\phi=0.00003$ that exhibits gaslike structure with negligible particle correlations. (B) $\ln \left[g^{(1)}(Q, t)\right]$ vs $Q^{2} t$ for the same suspension at three different scattering vectors.
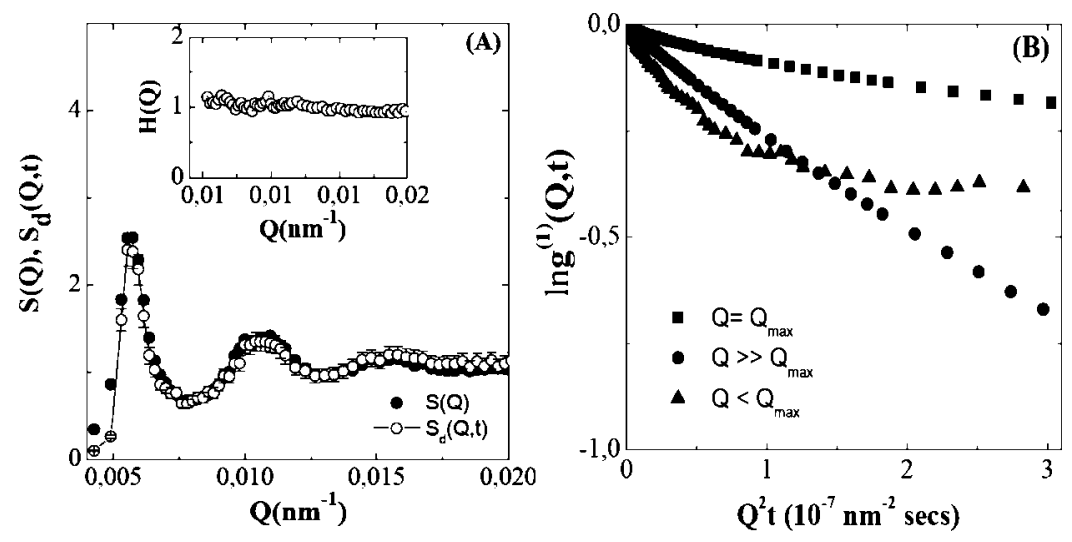

Figure 8. (A) Static structure factor $S(Q)$ vs $Q$ for a suspension (S2) with volume fraction of $\phi=0.001$ that exhibits the structural features of a strongly correlated liquid. The corresponding dynamic structure factor $S_{\mathrm{d}}(Q, t)$ is obtained from the ratio of the free diffusion coefficient $D_{\mathrm{o}}$ to the short-time diffusion coefficient $D_{\mathrm{s}}$. The inset shows the extracted hydrodynamic interaction $H(Q)$ as a function of $Q$. (B) $\ln \left[g^{(1)}(Q, t)\right]$ vs $Q^{2} t$ for the same suspension at $Q=Q_{\max }, Q<Q_{\max }$, and $Q \gg Q_{\max }$.
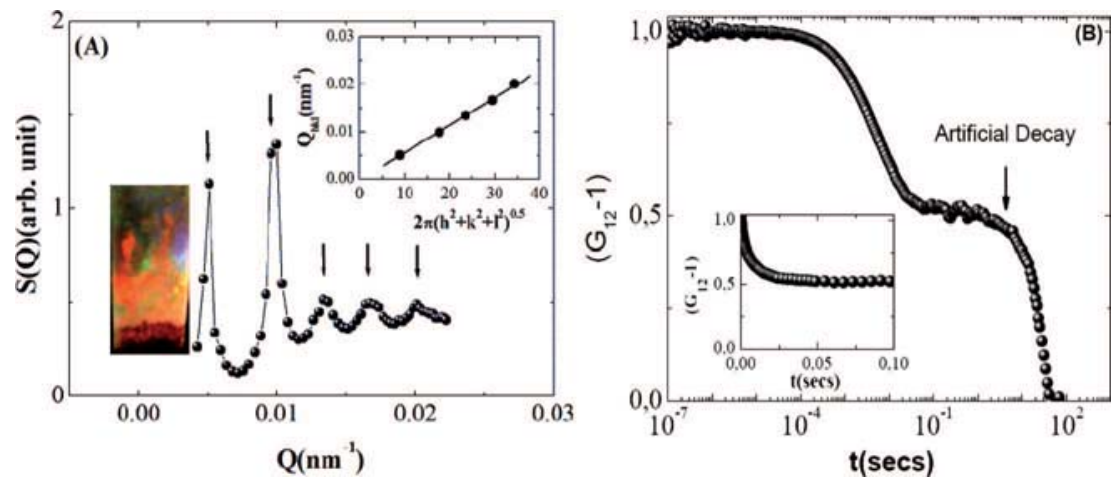

Figure 9. (A) $S(Q)$ as a function of the scattering vector $(Q)$ for a fully deionized crystalline suspension $\mathrm{S} 2$ at a volume fraction of 0.00074 . The peak positions were assigned to the (110), (220), (321), (332), and (521) reflections from a BCC crystal. The $Q_{h k l}$ values at these ( $\left.h k l\right)$ reflections are plotted as a function of $\left(h^{2}+k^{2}+l^{2}\right)^{0.5}$ and are shown as an inset. The photograph of the crystalline suspension is also shown as an inset. (B) Three-dimensional intensity cross-correlation function as a function of time. The measurement was carried out at the $Q=Q_{\max }$ position of the structure factor. The ensemble average is taken by rotating the sample cell slowly at $0.005 \mathrm{rpm}$, which leads to an artificial decay at correlation times larger than about $1 \mathrm{~s}$. The data has been normalized with respect to the intercept value of the measured correlation function. The inset shows the correlation function on a linear time scale with the artificial decay removed in order to illustrate the formation of a plateau in the correlation function due to the solidlike nature of the colloidal crystal.

a cumulant analysis of $g^{(1)}(Q, t)$ (eq 2) and using the relationship for the short-time diffusion coefficient $D_{\mathrm{s}}(Q)=\Gamma / Q^{2}$, we can then determine the dynamic structure factor $S_{\mathrm{d}}(Q, t)=D_{o} / D_{s}(Q)$. $S_{\mathrm{d}}(Q, t)$ is plotted in Figure 8A along with $S(Q)$. We observed good agreement between the dynamic and the static structure factor for all values of $Q$ (Figure 8A). This is not surprising for strongly correlated suspensions formed by highly charged particles at relatively low volume fractions that experience long-range weakly screened electrostatic repulsions. Under these conditions, hydrodynamic interactions are much less important than for concentrated suspensions, and the hydrodynamic function $H(Q)$ is expected to deviate only weakly from $1 .^{33}$ This is illustrated in the inset of Figure 8A where we plot $H(Q)$ calculated using $H(Q)=D_{\mathrm{s}}(Q) S(Q) / D_{\mathrm{o}}$ and find $H(Q) \approx 1$ at all $Q$ values, 
confirming the absence of strong hydrodynamic interactions under these conditions (inset of Figure 8A).

The crystalline suspension that formed after prolonged contact with the ion-exchange resin at a volume fraction of $\phi=$ 0.00074 showed iridescence due to the Bragg diffraction of visible light (inset of Figure 9A). Colloidal crystallites perpendicular to the scattering plane ( $X Y$ plane) were identified by observing the Bragg spots in the $X Y$ plane within the scattering volume. The obtained structure factor, $S(Q)$, plotted in Figure 9A shows five sharp Bragg peaks. These peaks positions are assigned according to the Bragg equation $Q_{h k l}=(2 \pi) /\left(a_{1}\right) \sqrt{ }\left(h^{2}+k^{2}+l^{2}\right)$, where $a_{1}$ is the lattice constant and $h, k$, and $l$ are the Miller indices of the Bragg diffraction plane. From the peak positions, the crystal structure was found to be of body-centered cubic (bcc) type, which is typical of highly charged colloids at low volume fractions. $Q_{h k l}$ shows a linearity with $\left(h^{2}+k^{2}+l^{2}\right)^{0.5}$ (shown as an inset in Figure 9A), and the lattice constant $a_{1}=$ $1730 \mathrm{~nm}$ was obtained from the slope. These core-shell particles with a highly charged corona of grafted polylectrolyte chains are thus capable of forming colloidal crystals at very low volume fractions.

In the next step, we then also investigated the dynamics of this crystalline suspension by measuring the intensity-intensity correlation function at the first peak position (at $Q=Q_{\max }$ ) of the structure factor. The sample is now solidlike and thus exhibits the classical features of so-called nonergodic systems with a vanishing long-time diffusion and the formation of a clear plateau in the correlation function, which therefore requires special measures in order to guarantee proper ensemble averaging. Therefore, the crystalline sample was ensemble-averaged by very slowly rotating the sample cell, which introduces an artificial decay in the correlation function that defines the baseline of the measurement and allows us to establish the height of the plateau correctly. The normalized intensity cross-correlation function $G_{12}(Q, t)$ clearly shows the formation of a plateau with a socalled nonergodicity parameter (height of the plateau) of about 0.5 (Figure 9B). In the inset of Figure 9B, the artificial cutoff decay from the slow sample rotation has been removed. The plateau in $G_{12}(Q, t)$ demonstrates that in the soft colloidal crystal the long-time diffusion is arrested and the sample shows solidlike behavior. In contrast to the strongly correlated liquidlike suspensions, the particles now can undergo only local excursions around their lattice site, which leads to arrested diffusive behavior and the formation of a plateau in the temporal evolution of the mean square displacement.

\section{Conclusions}

The strongly ionized amphiphilc block copolymer (poly(styrene)-block-poly(styrene sulfonate)) was found to be an efficient emulsifier for emulsion polymerization in the preparation of electrosterically stabilized colloidal particles of different diameters (between 70 to $400 \mathrm{~nm}$ ). Extensive characterizations of these particles showed that the synthesized particles are nearly monodisperse (polydispersity index $<6 \%$ ) as compared to previous studies on polymeric latex particles prepared by ionic amphiphilic block copolymers. A comparative study using dynamic light scattering in the solution state and transmission electron microscopy in the dried state confirmed that these colloidal particles are hairy in nature with the polymer chains stretched close to their full length at very low salt concentration. The obtained total dissociable charges and effective charges clearly suggested that most of the charges were confined to the area near the particle surface. Furthermore, these monodisperse colloidal particles self-assembled to form 3D-ordered photonic crystals even at volume fractions as low as $\phi=0.00074$. It is also expected that these electrosterically stabilized colloidal particles should be more stable with respect to added salt as compared to purely electrostatically stabilized colloids.

Acknowledgment. We gratefully acknowledge financial support by the Swiss National Science Foundation (grant number 200020-117755) and the Adolphe Merkle Foundation. This work is based in part on experiments performed at the Swiss spallation neutron source SINQ, Paul Scherrer Institute, Villigen, Switzerland. We are grateful for the neutron beam time, and we acknowledge the help of our local contact Ronny Vavrin. This work was also supported by the 21 st century COE program for a United Approach to New Materials Science from the Ministry of Education, Culture, Sports, Science and Technology, Japan. 